\section{Visual temporal integration and simple reaction time*}

\author{
MITCHELL L. KIETZMAN** and BARBARA J. GILLAM $†$ \\ Biometrics Research, New York State Department of Mental Hygiene \\ Brooklyn, New York 11203
}

Intensity-time reciprocity for simple RT to foveal pulses of light was demonstrated up to $11 \mathrm{msec}$ by using two experimental paradigms. The first paradigm was designed to separate two possibly confounded factors displayed by previous studies investigating the effects of increased stimulus duration on RT: (1) an asymptotic RT as a function of the increasing energy of a pulse as its duration is increased, and (2) the breakdown of integration as the pulse duration is increased. The second paradigm was designed to avoid the first factor so as to maximize the possibility of finding partial integration at long durations. In this paradigm, partial integration was demonstrated for additional light input presented as long as $64 \mathrm{msec}$ after stimulus onset. The failure of other studies to demonstrate temporal integration for $R T$ is discussed in terms of these paradigms.

Temporal integration in vision is demonstrated when a given response is determined by the luminous energy of the stimulus regardless of how that energy is distributed over time (stimulus intensity-time reciprocity). Numerous psychophysical investigations of temporal integration have been reported for a variety of response measures, such as absolute threshold (Bloch's law), differential threshold, acuity threshold, and wavelength and brightness discriminations (for examples, see Bartlett, 1965). There have been relatively few investigations of integration using response latencies, such as are measured in simple reaction time (RT) experiments, although Graham and Ratoosh (1962) have discussed the problem of relating visual $R T$ to temporal integration. They suggest that, up to a critical duration, the same RT should be obtained for either a brief light pulse of high luminance or a longer pulse of the same energy but lower luminance.

Theoretically, it would be possible to do a RT experiment that parallels psychophysical experiments of integration. In such a study, functions could be generated that depict the relationship between RT and the

*This research was supported by two grants (USPHS Grant MH-11688 and NSF Grant GB-5615). The data were collected and analyzed with the assistance of $E$. Shapiro. Equipment used in the experiments Shapiro, Equipment used in the experiments Laupheimer. Numerous help ful suggestions were provided by Dr. Harvey Babkoff during the investigation. The late $D r . N$. Beckenstein, former Director of Brooklyn State Hospital, provided space for the laboratory facilities.

**Also at the Department of Psychology, Queens College of the City University of New York.

+Now at the Department of Psychology, Columbia University. luminance of different, fixed-duration stimuli. These curves would enable the determination of the amount of stimulus energy necessary to produce a criterion RT and therefore would allow the determination of the characteristics of integration for RT. However, unlike psychophysical experiments where a constant criterion level of responding, such as a $50 \%$ detection level, has been previously established by tradition or theoretical considerations, there would be no guidelines as to which RT level to select for the response criterion. Therefore, for studies of response latencies, it seems necessary to develop procedures for measuring integration that are different from those that have been used in psychophysics.

In fact, existing visual RT studies of integration generally have not followed standard psychophysical procedures, since they involve varying the duration of a constant-luminance stimulus. In these experiments, the stimulus duration which produces an asymptotic RT has been referred to as critical duration (Raab \& Fehrer, 1962; Pease, 1964). However, in psychophysical experiments, critical duration usually is referred to as the termination of intensity-time reciprocity, and reciprocity cannot be only the duration of the stimulus is varied. The asymptotic RT obtained as a function of increased duration does not necessarily give an estimate of critical duration, as defined in psychophysics, for at least two reasons: first, the asymptotic RT may be attributable to the energy level of the stimulus and not to its duration per se, especially since RTs begin to asymptote at relatively low energy levels for very short duration visual stimuli (see Teichner, 1954); second, demonstrated in experiments in which there may be partial integration beyond critical duration, and therefore an asymptotic RT occurring as a function of increasing stimulus duration would not be at critical duration but at the end of partial integration. The termination of partial integration, or the end of any effect of increasing stimulus duration, is referred to here as "utilization time" following Piéron (1952).

The three experiments described in this report measured temporal integration for RT by using two stimulus paradigms that are distinct from those used either in psychophysical investigations of integration or in most previous RT experiments of integration. These designs allowed us to avoid the limitations of studies using the asymptotic RT estimates of critical duration and to measure all of the characteristics of temporal integration, including partial integration and utilization time.

\section{EXPERIMENT 1}

Experiment 1 was designed to measure integration by comparing the effects on RT of equivalent stimulus intensity and duration manipulations and not by merely manipulating stimulus duration. A similar experimental design for RT has been used by others (Kaswan \& Young, 1965; Bruder, 1971). In this experimental paradigm, critical duration can be separated from utilization time. The left column of Fig. 1 shows the two stimulus conditions employed, both of which measured $R T$ as a function of increasing energy. In the variable-luminance condition, the duration of the light pulse was held constant at 3.05 msec, and its luminance was varied between 0.12 and $2.06 \mathrm{~mL}$. In the variable-duration condition, the luminance of the pulse was held constant at $0.13 \mathrm{~mL}$ and its duration varied between 2.82 and 48.5 msec. $^{1}$

Stimulus conditions were chosen to provide for equal-paired products of duration times luminance, and these were designated Energy Level 1 (E1) through Energy Level 6 (E6). For example, in Fig. 1 at Energy Level 2, the variable-luminance stimulus is represented by a $0.27-\mathrm{mL}$ 3.05-msec flash and the variable-duration stimulus by a 0.13-mL 6.33-msec flash-both of which yield an energy product of $0.823 \mathrm{~mL}$-msec; Energy Level 5 is represented by two conditions yielding a product of $4.06 \mathrm{~mL}-\mathrm{msec}$. Under these conditions, intensity-time reciprocity would be demonstrated if the RTs to pairs of the equal-energy stimuli were the same, provided that 
EXPERIMENT 1

EXAMPLE

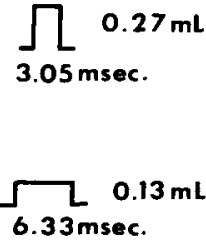

Energy Level 2 (E 2

EXAMPLE 2

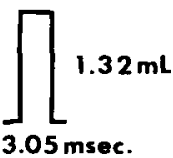

$0.13 \mathrm{~mL}$

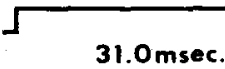

Energy Level 5 (E5)
EXPERIMENT 2

EXAMPLE
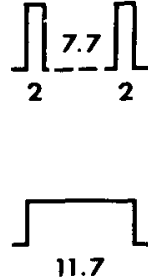

11.7

Total Duration $=11.7 \mathrm{msec}$.

EXAMPLE 2
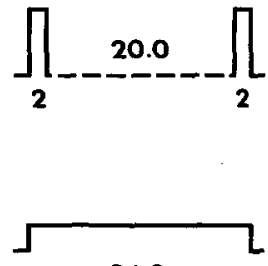

24.0

Total Duration $=24.0 \mathrm{msec}$.

HALF - ENERGY PULSE<smiles>C=CC</smiles>

Total Duration $=2.0 \mathrm{msec}$.
EXPERIMENT 3

EXAMPLE 1
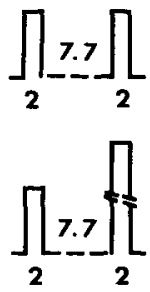

Total Duration $=11.7 \mathrm{msec}$.

EXAMPLE 2
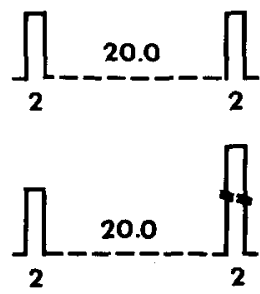

Total Duration = $24.0 \mathrm{msec}$.

HALF-ENERGY PULSE

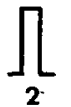

Total Duration $=2.0 \mathrm{msec}$.

Fig. 1. Stimulus conditions for Experiments 1, 2, and 3. Two examples of the stimulus variations are shown for each experiment.

these RTs decreased as the stimulus energy increased. The stimulus duration at which the RTs differ for a pair of equalenergy stimuli provides an estimate of critical duration; specifically, when longer RTs are obtained for points on the variable-duration curve than for the corresponding points on the variable-luminance curve, critical duration has been exceeded by the variable-duration stimuli and the longer duration stimuli are not fully integrated.

The interpretation of the RT asymptote of the variable-duration curve as an estimate of utilization time depends on the shape and level of the variable-luminance curve. If the variable-luminance curve also asymptotes for comparable energy values, then the absence of $R T$ changes for the variable-duration condition simply reflects the attainment of an RT asymptote due to the energy level of the stimulus and cannot be attributed to a failure of integration. Only if RT for the variable-luminance condition continues to decrease can the asymptote for the variable-duration condition be interpreted as an estimate of utilization time.

\section{Apparatus}

An image of the crater of a glow-modulator gas-discharge tube (Sylvania R1131C) was focused onto a diffusing glass producing a white-appearing circular target. An opaque mask on the glass outlined the target, which subtended a visual angle of $40 \mathrm{~min}$, viewed from a distance of 10.5 in. Foveal fixation was obtained by using four dim red lights surrounding the target. The glow-modulator tube was electronically gated. The durations of the light and dark times were controlled by a transistorized nine-channel multivibrator timer with an indeterminancy of 1 part in 10,000 (Logical Instruments Company). The rise and decay times of the glow-modulator light pulses were approximately 20 microsec and 2 microsec, respectively, at the current level used (23 mA). Chin- and foreheadrests were employed to obtain a stable position for $\mathrm{O}$.

\section{Method}

The Os were two male college students, who were paid for their services. Both had had between 25 and $30 \mathrm{~h}$ of prior e.rperience in RT experiments but were uninformed of the stimulus conditions or purpose of the experiment.

In each experimental session, there were six blocks of RT trials, one for each energy level. Each block of 14 trials consisted of a quasirandom presentation of variable-duration and variable-luminance pulses of the same total energy, so that 7 trials of each were obtained. The six blocks were given in a different random order during each of 11 sessions and yielded approximately 80 RTs per condition. There were $2-3$ sessions per day and a rest of at least $15 \mathrm{~min}$ between sessions.

\section{Procedure}

The $O$ was seated in a light-tight booth and was dark-adapted for $5 \mathrm{~min}$. On a signal from $E$ that a block of trials was about to begin, $O$ was required to place his head in position and to remain there until the end of the block. Each trial began with a warning click, which was a signal for $O$ to fixate. The click was followed, after a random interval of $1.5-2.5 \mathrm{sec}$, by the light pulse. The $O$ was instructed to lift his finger from a key immediately on seeing the flash. The interval between trials was approximately 8-10 sec. At the end of each block, $O$ was given a short rest in the booth.

Results

The median RTs of both Os for the variable-duration condition (squares and dashed lines) and the variable-luminance condition (circles and solid lines) are shown in Fig. 2. The brackets surrounding the data points represent the .05 confidence interval for each median. The range of semi-interquartile values (not shown) was 11.5-19.5 msec for O M.P. and 6.0-13.5 msec for O F.W., except that for both Os the semi-interquartile values were about 10.0 msec greater at the lowest energy level (E1).

Both Os gave similar results. As stimulus energy of the pulses of both conditions increased up to the E3 level, the RT curves decreased sharply and remained together, thus demonstrating a region of intensity-time reciprocity up to that level. Beyond E3, the two curves separated. The best estimate of critical duration for these data is approximately $11.0 \mathrm{msec}$, since the variable-duration $R T$ curve reached an 
asymptote around this level, whereas the variable-luminance RT curve continued to decrease slightly. This decrease indicates that the estimate of critical duration is valid, since the asy mptote of the variable-duration RT curve obviously was not due to the stimulus being at such a high energy level that the RTs could not be reduced further. Furthermore, since the variable-duration RT curve did not decrease for stimuli longer than the critical duration, i.e., beyond the E3 level, before reaching an asymptote, there is no evidence of partial integration. Therefore, for these stimulus conditions, it must be concluded that critical duration and utilization time are approximately the same and that the asymptotic RT provides an estimate of critical duration.

\section{EXPERIMENT 2}

In Experiment 2 an attempt was made to investigate the characteristics of integration for RT at a single energy level, i.e., energy was kept constant as stimulus duration increased and RT was measured at each duration. This stimulus paradigm provides a measure of temporal integration for RT in which stimulus energy does not increase with duration, as in Experiment 1, and therefore enables us to investigate further the possibility of partial integration. Two types of equal-energy stimuli were used: (1) a pair of 2-msec pulses of light of fixed luminance separated by varying interpulse intervals (a two-pulse condition similar to that used by Grossberg, 1970a); (2) single pulses of light in which luminance was decreased as duration increased (a one-pulse condition). RTs also were obtained for two other single-pulse stimuli. One was a 4-msec pulse with energy equal to the other equal-energy stimuli. (This stimulus was the same as the two-pulse stimuli, except that its interpulse interval was equal to zero.) The other was a 2-msec pulse of half the energy of all the other stimuli (the halfenergy pulse). It was identical to each of the pulses of the two-pulse stimulus condition.

The center figure of Fig. 1 (marked Experiment 2) shows the halfenergy pulse and two examples from the two-pulse and the one-pulse condition. All conditions are described in detail below.

In the two-pulse condition, intensity-time reciprocity is demonstrated when RTs to the two-pulse stimulus package remain the same as the RTs to the 4-msec pulse, even when the interpulse interval, and thus the total duration of the two-pulse stimulus, is increased. However, increases in stimulus

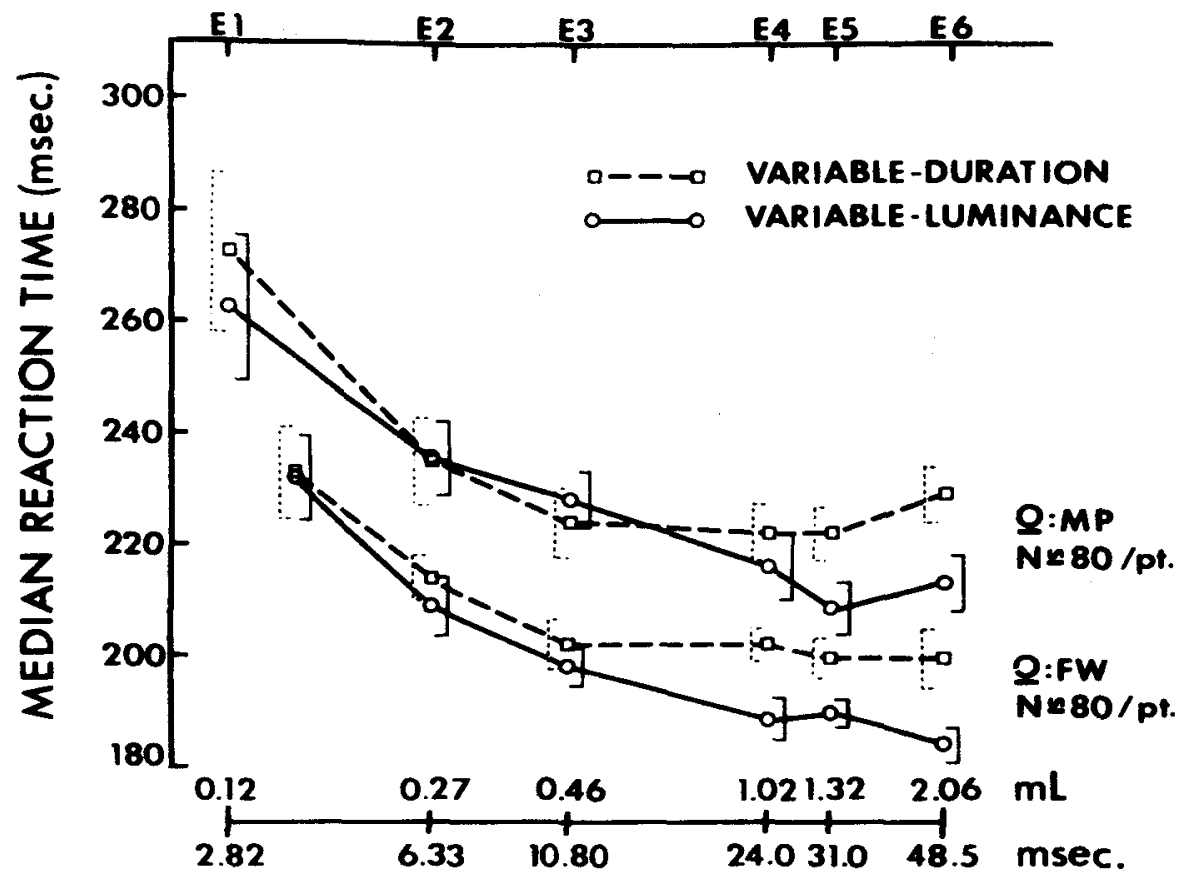

Fig. 2. Data of two Os showing changes in median RT as a function of increasing stimulus energy. At the different energy levels (E1, E2, ... E6), energy was obtained by varying either duration for a constant luminance or luminance for a fixed duration. The brackets surrounding the data points represent the .05 confidence limits.

duration should eventually produce longer RTs if the second pulse is not fully integrated. Critical duration is the total duration of the stimulus at which the RT first lengthens. Utilization time is the shortest stimulus duration that elicits an RT that is the same as the RT to the halfenergy pulse condition, since equal RTs would be predicted for these two conditions if none of the energy of the second pulse of the two-pulse stimulus were being integrated.

In the one-pulse condition, intensity-time reciprocity would be demonstrated by different duration stimuli producing the same RT. Critical duration is the point beyond which duration increments no longer compensate for luminance decrements and RT begins to increase. It is not possible to estimate utilization time for the one-pulse condition, since, unlike the two-pulse case, there is no RT measure that can be used as a baseline to indicate the end of energy integration.

The use of both one- and two-pulse conditions in the same experiment allowed us to compare RT measures of integration with the results of parallel psychophysical measures of integration. Kietzman and Sutton (1968) have shown that equal-energy one- and two-pulse stimuli of matched total durations are equally discriminable from a briefer comparison stimulus containing the same energy.

\section{Method}

RTs were measured from the onset of the first pulse of light and were obtained to the three kinds of stimuli described above (see Fig. 1): (1) Two-pulse stimuli-a stimulus package consisting of two pulses of light separated by interpulse intervals of $7.7,20.0,33.4,59.6$, and $77.8 \mathrm{msec}$ Additional interpulse intervals of 94.0 and $122.0 \mathrm{msec}$ were used for O F.W. Each pulse was $2.0 \mathrm{msec}$ in duration and was of a constant luminance $(0.18 \mathrm{~mL}$ for $O \mathrm{M} . \mathrm{P}$ and $0.15 \mathrm{~mL}$ for O F.W.). The two-pulse stimuli were specified with respect to their total duration, i.e., the 4.0-msec duration of the two pulses plus the duration of the interpulse interval. (2) One-pulse stimuli-a single-pulse stimulus with durations equal to the total durations of the two-pulse stimuli. The luminance values of these pulses were inversely proportional to the durations so that all single pulses had the same total energy as each other and as all the two-pulse stimuli. (3) Baseline stimuli-brief single pulses of light of $4.0 \mathrm{msec}$ and $2.0 \mathrm{msec}$. These stimuli were of the same luminance as the two-pulse stimuli. The energy of the 4.0-msec pulse was the same as that of 


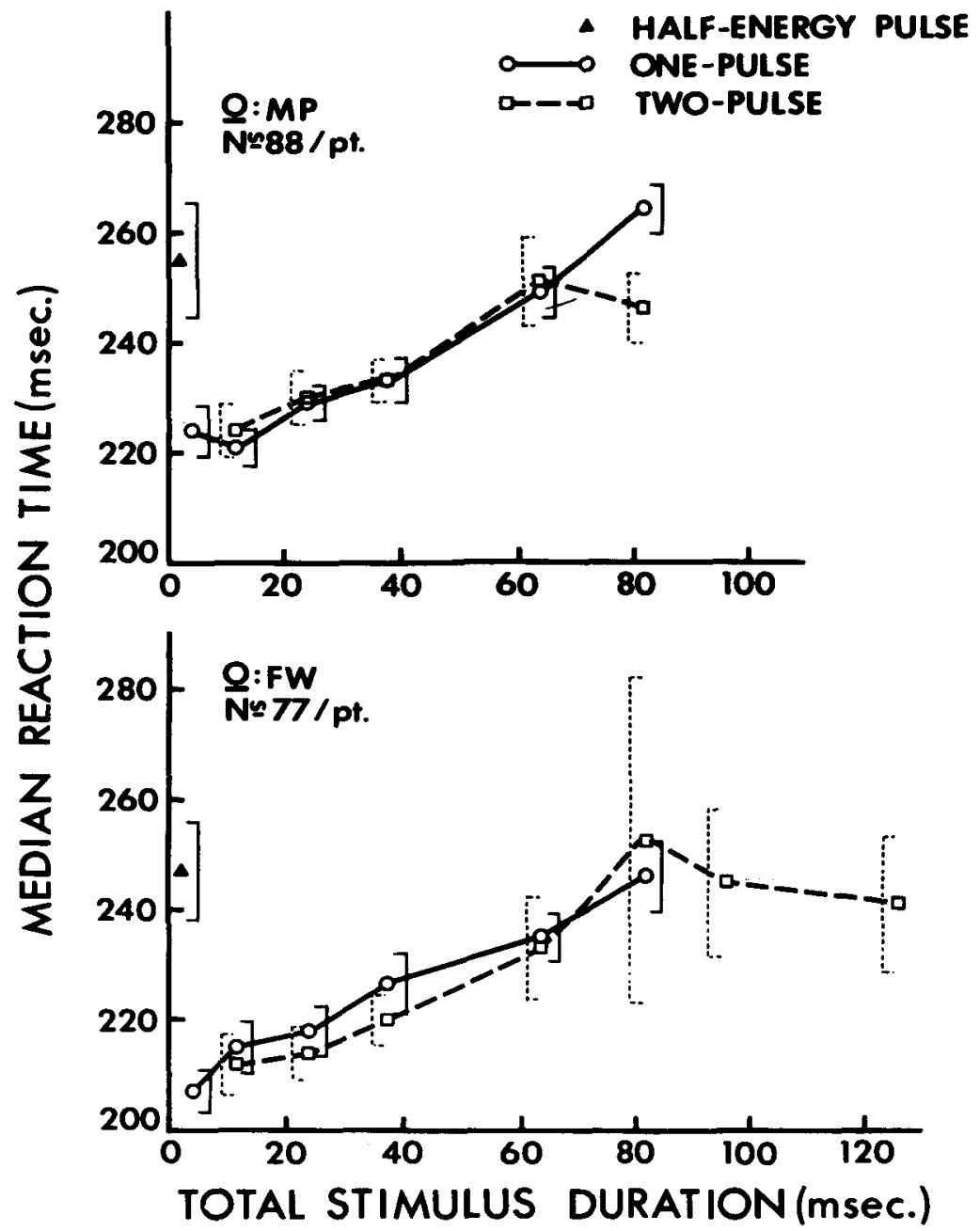

Fig. 3. Data of two Os (upper curve-O M.P., lower curve-O F.W.) showing changes in median RT as a function of the total duration of equal-energy stimuli packaged in two ways: (1) two-pulse stimuli of varying interpulse intervals ( $0--\square)$, or (2) one-pulse stimuli of increased duration and reduced luminance $(\circ \longrightarrow 0)$. The triangular points show data from the half-energy 2-msec condition. The brackets surrounding the data points represent the .05 confidence limits.

all stimuli in Conditions 1 and 2 , and the energy of the 2.0-msec pulse was half that of the other stimuli (half-energy pulse). These brief pulses were assumed to be fully integrated (the results from Experiment 1 support this assumption) and provided baseline data for estimating critical duration and utilization time.

Pilot experiments were conducted to establish a satisfactory luminance level for the 2.0- and 4.0 -msec pulses. The level chosen was the lowest luminance of a $2.0-\mathrm{msec}$ stimulus that could be detected $100 \%$ of the time (hence the slightly different values for Os M.P. and F.W.). ${ }^{2}$

In each experimental session, there was one block of 11 RT trials for each of 12 stimuli (14 stimuli for O F.W.). After each trial, feedback was given (by means of a tone) if the O's RT was faster than that of the previous trial.
The blocks were presented in a different random order during each session. The total number of RTs per stimulus condition was approximately 88 for O M.P. and 77 for O F.W.

\section{Results}

Median RTs for each condition are shown separately for each $O$ in Fig. 3. Confidence intervals at the .05 level are shown by the brackets surrounding each median. Semi-interquartile values (not shown) ranged from 8.0 to $31.0 \mathrm{msec}$ (one unexplainable exception of $68.0 \mathrm{msec}$ was obtained by $\mathrm{O}$ F.W. for the 81.8 -msec stimulus).

For both Os, as expected, there was a large RT difference between the 2and 4-msec stimuli. Complete intensity-time reciprocity for OM.P. two-pulse stimuli, as shown by the fact that the RTs were the same for pulses was obtained for both the one- and of 4.0 and $11.7 \mathrm{msec}$. This result agrees quite well with the estimate of $11 \mathrm{msec}$ for the critical duration for the same $O$ in Experiment 1. O F.W.'s data did not show unequivocally a region of intensity-time reciprocity.

Partial integration was demonstrated by the two-pulse data (squares and dashed lines) of both $\mathrm{Os,}$ as shown by the fact that RTs to the two-pulse stimuli did not reach the level of the RT to the halfenergy pulse until total stimulus durations of $63.6 \mathrm{msec}$ for O M.P. and $81.8 \mathrm{msec}$ for $O \mathrm{F.W}$. Up to these durations, the second pulse of light influenced the resulting $R T s$, since the first pulse alone could not have elicited such short reactions. Therefore, $64 \mathrm{msec}$ and $82 \mathrm{msec}$ are the best estimates of utilization time for these two Os.

Although the one-pulse condition cannot provide an estimate of utilization time, the one-pulse data (circles and solid lines) show evidence of partial integration. For example, the RT for OM.P. to the 63.6-msec one-pulse stimulus was equal to the RT obtained to the halfenergy pulse. Therefore, it can be concluded that half the energy in the 63.6-msec one-pulse stimulus was integrated. More than half the energy of each of the one-pulse stimuli of higher luminance and shorter duration than the 63.6-msec pulse was integrated, since RTs to these were shorter than RTs to the half-energy pulse.

The RTs to one- and two-pulse packages of identical stimulus durations were similar, especially for O M.P. Similar RTs for the two types of stimuli were anticipated if their stimulus durations were briefer than the critical duration, i.e., the stimuli were within the region of complete integration. The similar RTs for the one- and two-pulse stimuli that were obtained when the stimulus durations were longer than the critical duration, i.e., the stimuli were only partially integrated, indicated that the same amount of energy was integrated for these different stimuli. Apparently the RT response is not demonstrably sensitive to the distinctive energy distributions of these two types of stimuli.

\section{EXPERIMENT 3}

Experiment 3 was designed to measure and compare partial integration and utilization time for two sets of stimulus conditions: one in which the two pulses of light were of equal luminance (as in Experiment 2) and one in which the second pulse was of a higher luminance than the first pulse. 3

There were three stimulus


conditions (see Fig. 1), two of which were like the conditions in Experiment 2: (1) a 2.0-msec single pulse of light with a luminance of $.18 \mathrm{~mL}$ (the halfenergy pulse); (2) several two-pulse stimuli consisting of two 2-msec pulses separated by interpulse intervals of $0,7.7,20.0$, $33.4,46.0,59.6$, and 77.8 msec; (3) a new condition, consisting of two 2-msec pulse stimuli with the first pulse of each pair of the same luminance as the single pulse in Conditions 1 and 2 and the second pulse of a luminance $(1.54 \mathrm{~mL})$ that was eight and a half times greater than the luminance of the first pulse. Interpulse intervals for the unequal-luminance two-pulse stimuli were the same as for the equal-luminance stimulus of Condition 2.

Each of the 15 stimuli was presented in a random order once in each block. In all, there were approximately 88 blocks which gave 88 RTs per stimulus condition. Because of the randomization of stimuli, feedback could not be given. Data were obtained for O M.P., who had served in the previous experiments.

There were two phases in this experiment. The initial phase was the main portion of the experiment, and data collected for it are indicated by the filled points in Fig. 4. Data from a subsequent stage consisting of replication of some of the stimulus values plus some additional values (interpulse intervals of $63.6,81.0$, and $100 \mathrm{msec}$ ) are indicated by the unfilled points of Fig. 4.

\section{Results}

Median RTs for O M.P. for each condition are shown in Fig. 4. The confidence limits (.05) are indicated by brackets for each median. Semi-interquartile values (not shown) ranged from 8.0 to 23.5 msec. (Similar preliminary data based on a smaller number of trials, and therefore not shown, were obtained for another 0 .)

The data depicted in Fig. 4 show the same general trends for both the equal- and unequal-luminance pulses and are also similar to the results of Experiment 2. There is a large difference in RTs elicited by the 2-msec halfenergy pulse and the 4-msec pulse pair. As the total stimulus duration is increased, RT increases. For the longer stimuli, however, RT no longer increases but instead reaches an asymptotic value at about the same RT as that obtained to the half-energy pulse condition.

A comparison of the RTs obtained to the equal-luminance and the unequal-luminance conditions shows that all RTs are faster for the unequal luminance stimuli, a result that would

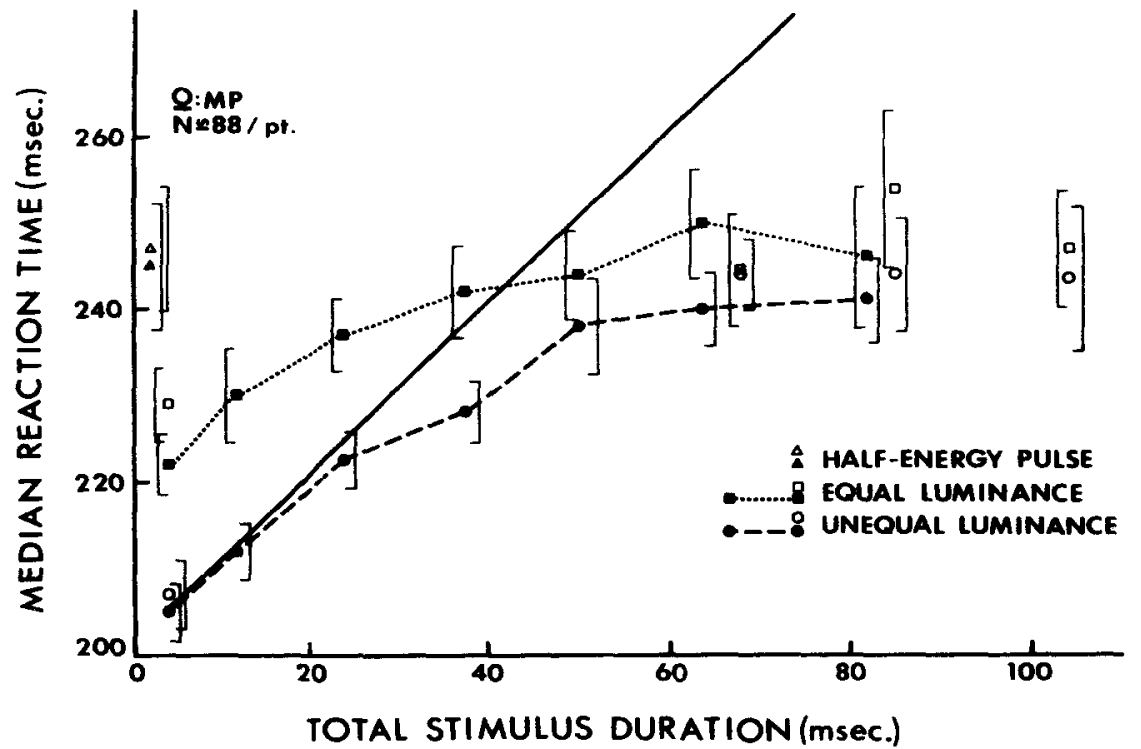

Fig. 4. Data of one $O$ showing changes in median RT as a function of increasing the interpulse interval between the two pulses of the stimulus package, thereby increasing the total stimulus duration. The upper curves (filled squares) are data from the condition in which the two pulses were of equal luminance; data of the lower curves (filled circles) are from the condition in which the luminance of the second pulse was 8.5 times more intense than that of the first pulse. The triangular points show data from the half-energy 2-msec condition. The unfilled data points are a replication and extension of the main experiment. The brackets surrounding the data points represent the .05 confidence limits.

be expected because of their greater energy. Both RT functions are similar in slope.

The major difference between the two functions of Fig. 4 is the stimulus duration at which the RT begins to asymptote, i.e., the estimate of utilization time. These data indicate that the utilization time of the two equal-luminance pulses (filled squares and dotted lines) was approximately 38 msec. ${ }^{4}$ The curve for the condition in which the second pulse had greater luminance than the first (filled circles and dashed lines) shows a utilization time that is longer than 50 msec; utilization time seemed longer when the luminance of the second pulse was greater.

The results for the unequal luminance pulses cannot be explained as a response to the second, higher-luminance pulse alone. The solid line in Fig. 4 represents hypothetical RTs that the 0 would have obtained if he had responded only to the second pulse. They were derived by adding each interpulse interval to the median RT obtained to two pulses with an interpulse interval of zero. In a pilot experiment, this condition yielded the same RT as did the second pulse presented alone. As can be seen in Fig. 4, RTs to the stimuli with total durations of 37.4 and $50.0 \mathrm{msec}$ were considerably faster than RTs to either the first or second pulse.

Figure 4 shows that the curve for the unequal-luminance pairs seems to asymptote at a faster RT level than does the curve for the equal-luminance pairs. In order to check this apparent difference, the second phase of the experiment was conducted using the half-energy pulse and both equal- and unequal-luminance pulse pairs at the longer interpulse intervals. In Fig. 4, the data from the second phase of the experiment (the unfilled points) show that the RTs for the two conditions asymptote at similar levels.

\section{DISCUSSION}

The experiments reported here describe two experimental paradigms to investigate the different characteristics of temporal integration as measured by simple RT. The results obtained from these paradigms indicate that for low-energy suprathreshold stimuli there is a region of intensity-time reciprocity as measured by simple RT, but this region is very brief, extending in these experiments only to about 11 msec. A recent comparable $R T$ finding by Bruder (1971) indicates intensity-time reciprocity for threshold-level stimuli as well, and this reciprocity extends for a slightly longer duration, up to about $\mathbf{3 0}$ msec. Bruder's experimental 
paradigm was the same as that used in Experiment 1. Pease (1971) also found a similar estimate of critical duration for RT (about $\mathbf{3 0} \mathrm{msec}$ ), using near-threshold stimuli in a two-pulse experimental paradigm similar to that used in Experiments 2 and 3. Taken together, these results indicate that Bloch's law does apply for simple RT, although the critical durations are very brief.

In most early psychophysical investigations of integration, the results were interpreted as displaying either complete integration or no integration. In their discussion of RT and integration, Graham and Ratoosh (1962), for example, state that above the critical duration, luminance alone would be expected to influence the response, i.e., above critical duration there is no integration. Recently, however, psychophysical studies have shown that there is an intermediate range of durations longer than critical duration where intensity-time reciprocity is absent but where changes in stimulus duration have some effect on the response; this range would be a region of partial integration (Baumgardt \& Hillmann, 1961; Kietzman \& Livingston, 1967; Sperling \& Jolliffe, 1965).

In Experiments 2 and 3, it was demonstrated that beyond critical duration there is a much longer period of time over which additional stimulus input can reduce the resulting $R T$ and that the amount of such a reduction systematically depended upon the time after the onset of the stimulus that the additional energy was presented. This region of partial integration can extend as long as 70-80 msec after the stimulus onset, which means that it may take up to one-third of the total RT.

Other RT studies also have displayed partial integration. For example, in the Grossberg two-flash study (1970a), the stimulus condition most comparable to those used in Experiment 2 (the $100 \%$ seen condition) gave an estimate of utilization time equal to 50 msec. Bruder (1971), working at a detection threshold level, found partial integration for RT out to at least 65 msec. We conclude that partial integration may be, under certain conditions, an important aspect of simple RT and that the RT is not necessarily completely determined in the first few milliseconds after the stimulus onset, as some have contended (Raab, Fehrer, \& Hershenson, 1961; Raab \& Fehrer, 1962).

Previous investigations have shown little reduction in $\mathrm{RT}$ as a function of increased stimulus duration and consequently have concluded that measures of response latency either do not follow Bloch's law or demonstrate little, if any, temporal integration (Grossberg, 1968; Kaswan \& Young, 1965; Raab \& Fehrer, 1962; Raab, Fehrer, \& Hershenson, 1961; Robinson, 1966). Several of these RT studies varied only the duration of the stimulus and used the asymptotic RT as an estimate of critical duration. However, there are two reasons why the asymptote may not be a suitable estimate of critical duration. The first is that, by equating the asymptotic RT with critical duration, the possibility of partial integration is not considered, and this experimental paradigm does not even allow partial integration to be measured. Usually, the asymptotic RT provides an estimate of utilization time, and the asymptotic RT would equal critical duration only in the case where there is no partial integration. In Experiment 1, for example, no partial integration was displayed and critical duration was equal to utilization time.

The second reason why the asymptote is not a suitable estimate of the critical duration (and perhaps why partial integration was not demonstrated in Experiment 1) is that the stimulus duration at which the RT first asymptotes may, in part, be attributable to the energy level of the stimulus and not to its duration. This problem may be understood by considering the slope of the function relating stimulus energy to $R T$. It is known that the RT-luminance function begins to decelerate at low luminance levels for pulses of brief duration. If RT were a function of energy irrespective of the time distribution of that energy, it follows that the RT-duration function must also asymptote at some duration for which the energy level of the stimulus is equivalent to the energy level of the asymptote point of the RT-luminance function. Therefore, an RT asymptote as a function of stimulus duration does not, in itself, demonstrate the end of integration. Those studies that have interpreted the asymptote in the RT-duration function to be a critical duration in the absence of any control data on the RT-luminance function for the energy levels used leave open the possibility that they were actually measuring, at least in part, an RT-energy asy mptote.

One of the most extensive of these studies (Raab \& Fehrer, 1962) does include data relevant to this problem, since duration was varied for each of five different luminance levels. Therefore, for any given duration, an RT-luminance function which gives an independent measure of the RT energy function can be found. Their data, replotted in this way, suggest that the shape of RT-nergy function may indeed account in some degree for their failure to find substantial duration effects on RT. Specifically, at the higher luminance levels of 30.0 , 300 , and $3,000 \mathrm{fL}$, they obtained mean RTs that differed by only 5-10 msec for each log unit difference in luminance. Since the total range of durations used in their study was only $1.6 \mathrm{log}$ units, it is likely that changes in RT as a function of duration would have been slight, even for conditions in which there was intensity-time reciprocity, and changes in RT for the region of partial integration would have been especially difficult to detect. At the lower luminance levels (below $30 \mathrm{fL}$ ) $\log$ unit differences in luminance were associated with larger differences in RT for brief duration pulses. It was only at these lower luminance levels that increasing the pulse duration led to a considerable decrease in RT and the RTs for these stimuli seemed still to be decreasing at $20 \mathrm{msec}$, the longest stimulus tested.

It is possible to help elucidate some of the seeming discrepancies between the present and previous investigations of integration for simple RT by comparing the measurement of temporal integration using the paradigms of Experiment 1, on the one hand, and Experiments 2 and 3 , on the other. The same $O$ (M.P.) was tested in Experiments 1, 2, and 3. In Experiments 2 and 3 , his data display considerable partial integration and a pulse presented as late as $65 \mathrm{msec}$ after the onset of the stimulus still reduced RT. In contrast, no partial integration was found in Experiment 1. Why? The fact that the variable-luminance control conditions of Experiment 1 elicited faster RTs beyond the energy at which critical duration occurred indicates that the RT asymptote as a function of stimulus duration was not attributable solely to the stimulus being at too high an energy level. The absence of partial integration may be attributable to the fact that even the R T reductions for the variable-luminance condition (pulses presumed to be fully integrated) are not large, probably because of the small changes in energy and the particular energy level of these stimuli. Thus, it is not surprising that the variable-duration curve beyond critical duration (pulses presumed to be only partially integrated) fails to show any reduction in RT. It is worth noting that Bruder (1971), using the same design as that of Experiment 1 but working at detection threshold levels of energy, found evidence of partial integration for durations perhaps as long as 90 msec. It is evident that this particular experimental paradigm, using very low energy stimuli, can be 
used to demonstrate partial integration.

These experiments indicate that in order to investigate temporal integration for RT comprehensively, at least two factors must be considered. First, it is important to use an appropriate experimental paradigm. For example, to demonstrate partial integration, one might use the two-pulse design of Experiments 2 and 3 , in which the stimulus energy is constant for different duration stimuli, thereby avoiding experimental paradigms that confound stimulus energy and duration. Second, it appears that temporal integration for $R T$ can only be demonstrated at low luminance and therefore at low energy levels (Pease, 1964) and that even for such data, an asymptote for RT as a function of increasing duration may reflect both changes in integration and the deceleration of RT as a function of increasing energy .

A broader view of Experiments 2 and 3 would be that there are at least three possible explanations for the faster RTs obtained to the two-pulse stimulus condition than to the half-energy one-pulse stimulus condition and for the changes that occurred as the interpulse interval between the pulses increased. First, the faster RTs could be due to the greater luminous energy presented in the two-pulse stimulus condition, and the changes in RT with interpulse interval increments could be due to the changes in integration of that stimulus energy. Second, the second pulse of the two-pulse stimulus may have a general activating effect on the $O$, which makes his RTs to the first pulse of light faster, and such an activation effect is time locked to the changes in the interpulse interval. Third, the two pulses in close temporal proximity provides $O$ on each trial with two stimuli to which he can respond, and his averaged $R T$ may be a statistical combination of the RTs to these individual pulses.

The first explanation in terms of temporal integration of luminous energy is, of course, the underlying interpretation of all the experiments reported here and is the interpretation used in previous RT investigations of visual integration (Grossberg, 1968, 1970a; Pease, 1971; Bruder, 1971). The second explanation in terms of a general activating effect that produces an RT facilitation has been offered and tested by Bernstein et al (1969, 1970). In his experiments, Bernstein obtained RTs to both a visual stimulus and to a visual-auditory stimulus pair with the visual stimulus presented first and varying interpulse intervals between the two stimuli. Bernstein obtained faster RTs to the double-pulse stimuli than to the light stimulus presented alone, and this RT facilitation decreased as the interpulse interval between the two stimuli increased. The third explanation in terms of statistical facilitation was offered by Raab (1962) to account for data obtained by Hershenson (1962) that were similar to those reported by Bernstein.

There are several reasons why we conclude that the results of Experiments 2 and 3 are best explained as due to temporal integration. One factor is the similarity in Experiment 2 between the one- and two-pulse RT functions and therefore the similarity in the characteristics of temporal integration. Since it is difficult to explain the one-pulse data in terms of either a general activation effect, similar to that described by Bernstein, or as due to statistical facilitation, similar to that described by Raab, the most parsimonious explanation of the similarity of these functions would seem to be that both are due to parallel changes in the integration of luminous energy.

Second, it is important to note that both the activation and the statistical facilitation explanations have arisen from experiments employing double-pulse stimulus conditions from different modalities, e.g., a visual stimulus and a subsequent auditory stimulus. Under these conditions, Bernstein's interpretation of facilitation as due to activation seems particularly applicable, since, other things being equal, auditory RTs are faster than visual RTs (Woodworth \& Schlosberg, 1954) and an auditory pulse with a shorter latency could serve as an alerting stimulus to reduce the visual RT even when presented after the visual stimulus. However, in our experiments, with the second pulse always being a visual stimulus, especially in Experiment 2 where the intensity of the second pulse is equal to that of the first pulse, it is difficult to see how the second pulse could serve as an alerting stimulus to facilitate a reaction to a previously presented stimulus.

We have obtained (unpublished data) evidence of the same type of facilitation reported by Bernstein by presenting an auditory stimulus at brief intervals after the first pulse of our two-pulse stimulus. The effect was to reduce RTs to all stimuli with the auditory alerting pulse, but the shape of the functions for the two-pulse stimuli with and without the auditory pulse were the same, i.e., there was no effect upon the characteristics of temporal integration.

To test the possibility of some type of statistical facilitation in our two-pulse experiments, we analyzed the data by a method similar to that employed by Raab (Gibbon, personal communication). This analysis showed that our obtained RTs, unlike those obtained by Hershenson (1962), cannot be explained simply in terms of the summation of two distinct RT distributions, one for each of the two pulses presented. Furthermore, the luminance level of the pulses and the brief interpulse intervals used in our experiments were such that most, if not all, of the two-pulse stimuli were not seen as two distinct flashes of light (Kietzman \& Sutton, 1968). Therefore, it seems unlikely that a "two-look" hypothesis could account for these data, although such a hypothesis would seem much more tenable for the Hershenson and Bernstein studies which used different modality stimuli and longer interpulse intervals.

\section{REFERENCES}

BARTLETT, N. R. Thresholds as dependent on some energy relations and characteristics of the subject. In $C$. $H$. Graham (Ed.), Vision and visual perception. New Yodk: Wiley, 1965.

BAUMGARDT, E., \& HILLMANN, B. Duration and size as determinants of peripheral retinal response. Journal of the Optical Society of America, 1961, 51, 340-344.

BERNSTEIN, I. H., ROSE, R., \& ASHE, V. M. Energy integration in intersensory facilitation. Joumal of Experimental Psychology, 1970, 86, 196-203.

BERNSTEIN, I. H., CLARK, M. H., \& EDELSTEIN, B. A. Effects of an auditory signal on visual reaction time. Journal of Experimental Psychology, $1969,80,567-569$.

BRUDER, G. E. Temporal integration for reaction time at threshold levels of luminous energy. Paper presented at the meeting of the Eastern Psychological Association, New York, April 1971.

GRAHAM, C. H., \& RATOOSH, P. Notes on some interrelations of sensory psychology, perception, and behavior: Rational accounts of sensory relations. In S. Koch (Ed.), Psychology: A study of a science, Vol. 4. Biologically oriented fields: Their place in psychology and in biological science. New York: McGraw-Hill, 1962.

GROSSBERG, $M$. The latency of response in relation to Bloch's law at threshold. Perception \& Psychophysics, 1968, 4. 229-232.

GROSSBERG, M. Frequencies and latencies in detecting two-flash stimuli. Perception \& Psychophysics, 1970a, 7, 377-380.

GROSSBERG, M. "Back ward masking" of simple detection latencies. Perception \& Psychophysics, 1970b, 8, 308-312.

HERSHENSON, M. Reaction time as a measure of intersensory facilitation. Journal of Experimental Psychology, $1962,63,289-293$.

KASWAN, J., \& YOUNG, S. Effect of luminance, exposure duration, and task complexity on reaction time. Journal of Experimental Psychology, 1965, 69, $393-400$.

KIETZMAN, M. L.. \& LIVINGSTON, D. Bloch's law for suprathreshold stimuli: A quantitative estimate of partial integration. Paper presented at the meeting of the Psychonomic Society, Chicago, October 1967.

KIETZMAN, M. L., \& SUTTON, S. The interpretation of two-pulse measures of 
temporal resolution in vision. Vision Research, 1968, 8, 287-302.

MURRAY, H. G. Stimulus intensity and reaction time: Evaluation of a decision-theory model. Journal of Experimental Psychology, 1970, 84, 383-391.

PEASE, V. P. The intensity-time relation of a stimulus in simple visual reaction time. Psychological Record, 1964, 14, 157-164.

PEASE, $V$. P. The effects of luminance and the duration of flash interval on simple visual reaction time. Paper presented at the meeting of the Eastern Psychological Association, New York, April 1971.

PIERON, $M$. The sensations. (Trans. $M$. $H$. Pirenne and B. C. Abbott.) New Haven: $Y$ ale University Press, 1952 .

RAAB, D. H. Statistical facilitation of simple reaction times. Transactions of the New York Academy of Sciences, 1962 . 24, 574-590.

RAAB, D. H. \& FEHRER, E. The effect of stimulus duration and luminance on visual reaction time. Journal of Experimental Psychology, 1962, 64, 326-327.

RAAB , D. H. FEHRER, E. \& HERSHENSON, M. Visual reaction time and the Broca-Sulzer phenomenon. Journal of Experimental Psychology, $1961,61,193-199$

ROBINSON, D. N. Visual reaction time and the human alpha rhythm: The effects of stimulus luminance, area, and duration. Joumal of Experimental Psychology. $1966,71,16-25$.

SPERLING, H., \& JOLLIFFE, C. L Intensity-time relationship at threshold for spectral stimuli in human vision. Journal of the Optical Society of America, 1965, 55, 191-199.

THRANE, V. C. Sensory and preparatory factors in response latency. II. Simple reaction or compensatory interaction? Scandinavian Journal of Psychology, $1960,1,169-176$

TEICHNER, W. H. Recent studies of simple reaction time. Psychological Bulletin $1954,51,128-149$

WOODWORTH, $R$, $\mathbf{S}$ \& SCHLOSBERG, $H$ Experimental psychology. (Rev, ed.) New York: Holt, 1954 .

\section{NOTES}

1. For O F.W. the $0.13-\mathrm{mL}$ pulse was below threshold at $2.82 \mathrm{msec}$. A pulse of $3.66 \mathrm{msec}$ was substituted, and the luminance of the corresponding 3.05-msec pulse increased to $0.16 \mathrm{~mL}$ to make the two stimuli equal in energy.

2. The luminance level selected by this criterion resulted in large RT differences between the 2- and 4-msec pulses, which suggested that the luminance manipulation used for the one-pulse condition were not asymptotic for RT. The results of Experiment 1 provide further evidence concerning this point. The variable-luminance curve from Experiment 1 shows that decreasing energy from $\mathbf{E 2}$ (0.83 mL-msec) greatly increases RT. Since the energy of the double pulses in Experiment 2 (0.72 and $0.62 \mathrm{~mL}$-msec for Experiment 2 (0.72 and $0.62 \mathrm{~mL}-\mathrm{msec}$ for
Os M.P. and F.W., respectively) were less than this energy level, RT should be highly sensitive to a breakcown of complete integration of these pulses. Therefore, if constant RTs were obtained under these constant RTs were obtained under these
low-luminance conditions, intensity-time reciprocity could be inferred.

3. Grossberg (1970b) recently reported a similar RT experiment using unequal luminance stimuli. The results he obtained were like those reported here, but he interpreted them in terms of visual masking rather than in terms of temporal integration.

4. The shorter estimate of utilization time obtained in Experiment 3 than that obtained in Experiment 2 for the equal-luminance pulses may be due to the complete random programming of stimuli in Experiment 3; in Experiment 2, the stimulus durations were blocked. For a discussion of the effects of programming on RT, see Murray (1970) and Thrane (1960).

(Accepted for publication January 10 , 1971.) 